\title{
Immunoglobulin G4-positive ascending thoracic aortitis may be prone to dissection
}

Henri Kajander, MB, ${ }^{\mathrm{a}}$ Timo Paavonen, MD, PhD, ${ }^{\mathrm{a}}$ Timo Valo, MB, ${ }^{\mathrm{a}}$ Matti Tarkka, MD, PhD, ${ }^{\mathrm{b}}$ and Ari A. Mennander, MD, $\mathrm{PhD}^{\mathrm{b}}$

Objective: Immunoglobulin (Ig) G4-positive aortitis may determine outcome after surgery for ascending aorta. We evaluated IgG4 expression of dilated ascending aortic wall.

Methods: The study consisted of 91 patients who underwent ascending aortic surgery. For histology, hematoxylin-eosin, elastase-van Gieson, and periodic acid-Schiff stainings were performed. The amount of $\mathrm{T}$ and B lymphocytes, plasma cells, macrophages, cell proliferation, and IgG4 positivity were determined by immunohistochemistry.

Results: The aortic wall in 12 patients had IgG4 positivity that was always confined to the adventitia. Adventitial plasma cells were numerous in all but 2 of these patients $(P<.0001)$. Aortitis was revealed in 2 patients $(17 \%)$ with IgG4-positive staining of the aorta and in 6 patients $(8 \%)$ with IgG4 negativity. IgG4 staining was significantly associated with total aortic wall inflammation (area under the curve, 0.865; standard error, 0.043; $P=.000 ; 95 \%$ confidence interval, 0.779-0.950). The mean diameter of the ascending aorta was $69 \pm 4.7 \mathrm{~mm}$ and $56 \pm 1.1 \mathrm{~mm}$ in patients with IgG4 positivity and negativity, respectively $(P<.004)$. Approximately half of the patients with IgG4 positivity had dissection $(42 \%)$, compared with only 15 of $79(19 \%)$ of the remaining patients $(P=$ not significant). Two patients with IgG4 positivity had to undergo reoperation because of immediate postoperative dissection. Seven patients died, including 4 patients (33\%) with IgG4 positivity; the remaining 3 patients $(4 \%)$ were IgG4 negative $(P<.005)$.

Conclusions: IgG4-positive ascending aortic wall was frequent in our study cohort (13\%) and revealed aortic inflammation associated with dilatation. (J Thorac Cardiovasc Surg 2013;146:1449-55)

The degree of ascending aortic wall inflammation may determine outcome after surgery. The diagnosis of inflammation is fundamental because aortitis and ascending aortic dissection $(\mathrm{AD})$ are associated with increased mortality. ${ }^{1-3}$ Intensive histopathologic studies of the ascending aorta have recently opened discussion for the clinical significance of aortitis, such as giant cell aortitis and idiopathic aortitis, to name but a few definitions of inflammatory and noninfectious isolated thoracic aortitis. ${ }^{4,5} \mathrm{~A}$ burden of variations in the histopathologic

\footnotetext{
From the Department of Pathology, ${ }^{\text {a }}$ Fimlab Laboratories, Tampere University Hospital and Tampere University; and Heart Center, Cardiac Research, ${ }^{\mathrm{b}}$ Tampere University Hospital, Tampere, Finland.

Funding: The Competitive Research Foundation of Tampere University Hospital (Grant 9L058), Tuberculosis Foundation, The Finnish Heart Association, and The Finnish Cultural Foundation. Dr Mennander is the recipient of the Ingegeerd and Viking O. Bjork award for Scandinavian Cardiovascular Research.

Disclosures: Authors have nothing to disclose with regard to commercial support.

Presented at the Aortic Symposium 2012, New York, New York, April 26-27, 2012, as part of the presentation on demand part of the meeting.

Received for publication March 18, 2012; revisions received July 4, 2012; accepted for publication Sept 13, 2012; available ahead of print Oct 11, 2012.

Address for reprints: Ari A. Mennander, MD, PhD, Heart Center, Cardiac Research, Tampere University Hospital, SDSKIR, Teiskontie 35, PL 2000 Tampere, Finland (E-mail: ari.mennander@pshp.fi).

$0022-5223 / \$ 36.00$

Copyright (c) 2013 by The American Association for Thoracic Surgery

http://dx.doi.org/10.1016/j.jtcvs.2012.09.039
}

features of patients with ascending aortic dilatation (AAD) is reported. ${ }^{6,7}$ Recent attempts to elucidate the association of inflammation with $\mathrm{AAD}$ and $\mathrm{AD}$ have generated theories on activation of aortic wall inflammation together with aortic wall remodeling. ${ }^{8,9}$ The fundamental question to the clinician is to identify new tools to help in decisionmaking concerning resection for AAD.

Immunoglobulin (Ig) G4-positive aortitis has recently been introduced to the literature of isolated thoracic aortitis. ${ }^{10-12}$ Only a few articles are available describing a highly rare IgG4-related sclerosing disease ${ }^{12}$ or IgG4related systematic disease of the aorta ${ }^{10,11,13}$ that accounted for $9 \%$ of cases of noninfectious isolated thoracic aortitis. The definition criteria are proposed to strictly include at least $50 \%$ presence of positively stained plasma cells for IgG4 positivity. ${ }^{13}$ Of note, the association of $\mathrm{AD}$ with IgG4-positive aortitis has been proposed, ${ }^{11}$ but no investigators have confirmed this. ${ }^{12}$

We systematically evaluated aortic wall inflammation in a series of consecutive patients who underwent operation for AAD and sought for IgG4 positivity. First, we investigated the inflammatory aortic wall profile. Second, we sought for clinical relevance of IgG4 positivity, including the likelihood of increased AAD, increased frequency of $\mathrm{AD}$, and early outcome compared with IgG4-negative patients. 


$$
\begin{aligned}
& \text { Abbreviations and Acronyms } \\
& \begin{aligned}
\text { AAD } & =\text { ascending aortic dilatation } \\
\text { AD } & =\text { aortic dissection } \\
\text { AUC } & =\text { area under the curve } \\
\text { CI } & =\text { confidence interval } \\
\text { Ig } & =\text { immunoglobulin } \\
\text { PSU } & =\text { point score units } \\
\text { ROC } & =\text { receiver operating characteristic } \\
\text { SE } & =\text { standard error } \\
\text { STJ } & =\text { sinotubular junction }
\end{aligned}
\end{aligned}
$$

\section{MATERIALS AND METHODS}

\section{Study Protocol and Surgery}

After institutional review board approval, ascending aortic wall resection of 101 consecutive patients undergoing surgery for ascending aorta was obtained and processed for histology. AAD was preoperatively confirmed and evaluated with computed tomography. According to our institutional policy, AAD included an aortic diameter more than $5.5 \mathrm{~cm}$ wide or aortic growth more than $1 \mathrm{~cm}$ in 1 year. This definition was adjusted to the presence of Marfan syndrome, gender, patient size, and symptoms including AD, according to the Yale Center criteria. ${ }^{14}$ Surgery was performed between July 2007 and September 2009 at the Heart Center of Tampere University Hospital, and all cases of AAD including AD processed for histology were enrolled. The samples of 10 patients were excluded because of technical failure during preparation of the samples.

The decision on the extension of resection and surgical technique was at the discretion of the operating surgeon. When AAD including the sinotubular junction (STJ) was estimated as the reason for aortic regurgitation, the STJ was tailored for a suitable graft in a supracoronary fashion. Whenever dilatation included the aorta root, a radical resection of the dilated ascending aorta together with the root and the aortic valve was performed in all but 4 patients, in whom a David-type valve-sparing operation was performed. The graft size was estimated by the principal surgeon. Resection of the aortic arch was carried out depending on extension of distal aortic dilatation. Because the surgical procedure was performed according to the surgical decision, the sample was procured from the middle of the resected diseased area of the ascending aorta in the vicinity of the STJ.

\section{Histology and Immunochemistry}

Up to 5 blocks of resected ascending aorta were embedded in paraffin, cut into $4-\mu \mathrm{m}$-thick segments, and stained with hematoxylin-eosin, Verhoeff-van Gieson, elastase-van Gieson, and periodic acid-Schiff. A representative $1-\mathrm{cm}-$ long piece of ascending aortic wall corresponding to all different staining was evaluated systematically for all resected samples procured during surgery. The heights of various layers (adventitia, media, and intima) were calculated for each sample. Inflammatory cells, intensity of inflammation, medial degeneration, intima cellularity, and thickness were estimated as previously described and expressed as point score units (PSU). ${ }^{9}$ For semiquantitative analyses, inflammation was graded as none, mild, moderate, or severe $(0,1,2$, or 3 , respectively). Medial degeneration was graded as patchy, moderate, or severe again on a scale of 0 to 3 . Intima cellularity and thickness were estimated according to an arbitrary scale from 0 to 3 , where 0 indicated normal intima with a single endothelial cell layer; 1 indicated intima cellularity and thickness less than $25 \%$ compared with the media; 2 indicated intima cellularity and thickness more than $25 \%$ but less than $50 \%$ compared with the media; and 3 indicated intensive intima cellularity and thickness more than $50 \%$ compared with the media.

Immunohistochemistry was performed using Ventana Lifesciences Benchmark XT Staining module (Ventana Medical Systems, Tucson,
Ariz). ${ }^{9}$ IgG4 (dilution 1:85) (binding site) was stained for slides accordingly. Ventana Lifesciences Antibody Dilution Buffer was used for dilution media. The samples were blindly evaluated for each primary antibody from the intima, media, and adventitia layers separately and categorized on a scale of 0 to 3 and expressed as PSU by 4 authors (H.K., T.V., T.P., A.M.), and 5 fields $(\times 40)$ were arbitrarily reviewed for each $1-\mathrm{cm}-$ long aortic sample. If patchy lesions of the aortic wall were identified, we chose the field area including the respective media, intima, and adventitia layers as mapped according to the thickest intima layer that coincided with positive IgG4 staining. Calculated adventitial inflammation was the sum of inflammatory cell staining of Tand B cells, macrophages, and plasma cells. Total aortic wall inflammation was obtained by adding the sum of inflammatory cell staining of intima, media, and adventitia.

\section{Statistical Analysis}

Whenever IgG4-positive staining was identified, it was always found in the adventitia and often the media. IgG4 positivity occasionally was observed in the intima. To determine the clinical relevance associated with histology, the patients were divided into 2 groups according to the presence of adventitial IgG4-positive staining of the aortic wall. Quantitative variables were listed as the mean and standard error (SE) of the mean. Categoric variables were stated as the count and percentage. Statistical analysis was performed with the Statistical Package for the Social Sciences version 19.0 (SPSS Inc, Chicago, Ill). The Mann-Whitney test was used for continuous variables, and the chi-square test was used for categoric analysis. Aortic wall inflammation and calculated adventitial inflammation were tested for correlation by Spearman rank rho. The predictive value of IgG4 positivity to identify aortic wall inflammation among patients undergoing surgery for ascending aorta was assessed by receiver operating characteristic (ROC) curve analysis.

\section{RESULTS \\ Patient Demographics}

The majority of the patients were male, and one third were female. The mean diameter of the ascending aorta at STJ was $57 \pm 1.1 \mathrm{~mm}$ for all patients, but was clearly larger in patients with IgG4 positivity as observed in patients with IgG4 negativity $(69 \pm 4.7 \mathrm{~mm}$ and $56 \pm 1.1 \mathrm{~mm}$, respectively, $P<.004)$. Approximately half of the patients with IgG4 positivity had $\mathrm{AD}(42 \%)$ compared with only 15 of $79(19 \%)$ of the rest of the patients $(P=.079)$. The mean age of the patients was $62 \pm 1.4$ years. There were 5 patients $(6 \%)$ with Marfan syndrome, of whom 4 had IgG4 negativity. Hypertension was diagnosed in only 27 patients, and no difference in its prevalence between the groups was found. The 1 patient with a nonspecific vasculitis in our study group was also IgG4 negative. The frequency of preoperative symptoms relative to inflammatory state, such as respiratory distress and arthritis, showed no difference between the groups. Moderate to severe aortic valve insufficiency was found in 40 patients, and aortic valve stenosis was found in 29 patients. The majority of patients had tricuspid aortic valves. One patient with IgG4 positivity and 6 patients with IgG4 negativity underwent a previous cardiothoracic operation (Table 1).

\section{Operative Technique}

Graft replacement for the ascending aorta was performed with root replacement (in 51 patients) or without 
TABLE 1. Patient demographics

\begin{tabular}{|c|c|c|c|c|}
\hline & All patients & IgG4+ & IgG4 & $P$ value \\
\hline No. of patients & $91(100 \%)$ & 12 & 79 & \\
\hline $\begin{array}{l}\text { Mid-ascending aorta } \\
\text { diameter, mm }\end{array}$ & $57 \pm 1.1$ & $69 \pm 4.7$ & $56 \pm 1.0$ & $<.004$ \\
\hline Dissection, $\mathrm{n}(\%)$ & 20 & 5 & 15 & NS \\
\hline Age (y) & $62 \pm 1.4$ & $63 \pm 4.6$ & $62 \pm 1.5$ & NS \\
\hline Male, $n(\%)$ & $63(69 \%)$ & 9 & 54 & NS \\
\hline Respiratory distress, n (\%) & $3(4 \%)$ & 1 & 2 & NS \\
\hline Marfan, n (\%) & $5(6 \%)$ & 1 & 4 & NS \\
\hline Hyperthyreosis, n (\%) & $4(5 \%)$ & 1 & 3 & NS \\
\hline $\begin{array}{l}\text { Central nervous } \\
\text { instability, n }(\%)\end{array}$ & $7(8 \%)$ & 1 & 3 & NS \\
\hline Myasthenia, n (\%) & $1(1 \%)$ & 1 & 0 & NS \\
\hline Hypertension, n (\%) & $27(30 \%)$ & 4 & 23 & NS \\
\hline Arthrosis, arthritis, n (\%) & $12(13 \%)$ & 1 & 11 & NS \\
\hline $\begin{array}{r}\text { Myocardial coronary artery } \\
\text { disease, infarction, } \mathrm{n}(\%)\end{array}$ & $16(18 \%)$ & 3 & 13 & NS \\
\hline Vasculitis, n (\%) & $1(1 \%)$ & 0 & 1 & NS \\
\hline $\begin{array}{l}\text { Previous cardiothoracic } \\
\text { operation }\end{array}$ & & & & NS \\
\hline $\begin{array}{l}\text { Coronary artery bypass } \\
\text { surgery, } \mathrm{n}(\%)\end{array}$ & $5(6 \%)$ & 1 & 4 & \\
\hline $\begin{array}{l}\text { Correction of aortic } \\
\text { coarctation, } \mathrm{n}(\%)\end{array}$ & $2(2 \%)$ & 0 & 2 & \\
\hline \multicolumn{5}{|l|}{ Aortic valve insufficiency } \\
\hline Moderate to severe, $\mathrm{n}(\%)$ & $40(44 \%)$ & 5 & 36 & NS \\
\hline \multicolumn{5}{|l|}{ Aortic valve stenosis } \\
\hline Moderate to severe, $\mathrm{n}(\%)$ & $29(32 \%)$ & 1 & 28 & NS \\
\hline
\end{tabular}

encompassing the root (in 40 patients). The extension of root dilatation together with dilatation of the ascending aorta from STJ was present in 47 patients $(60 \%)$ with IgG4 negativity in contrast to only 34 patients $(33 \%)$ with IgG4 positivity ( $P=$ not significant). A valve-sparing David operation was offered to 4 patients. Concomitant coronary artery bypass grafting was required by 12 patients (Table 2 ).

\section{Postoperative Findings, Histology, and Immunohistochemistry}

Confirmed by histology, aortitis with IgG4-positive staining was revealed in 2 patients $(17 \%)$ and 6 patients $(8 \%)$ with IgG4 negativity $(P=$ NS). Two patients with IgG4 positivity and none with IgG4 negativity had to undergo reoperation because of immediate postoperative $\operatorname{AD}(P<.05)$. Although $\mathrm{AD}$ was confirmed histologically, in 6 patients ( 2 with IgG4 positivity and 4 with IgG4 negativity), the adventitia was difficult to evaluate for each inflammatory cell in every sample block because of blood stains and artifact. Seven patients died, including $4(33 \%)$ with IgG4 positivity; the remaining 3 patients $(4 \%)$ were $\operatorname{IgG} 4$ negative $(P<$ $.005)$. Three patients with dissection and IgG4 positivity underwent operation without root surgery and died immediately after weaning from cardiopulmonary bypass as the
TABLE 2. Operative details according to surgical evaluation of extension of diseased aorta

\begin{tabular}{|c|c|c|c|c|}
\hline & All patients & IgG4+ & IgG4 & $P$ value \\
\hline & $91(100 \%)$ & 12 & 79 & \\
\hline $\begin{array}{c}\text { Graft replacement of root } \\
\text { and ascending aorta }\end{array}$ & & & & NS \\
\hline Mechanical conduit & $33(36 \%)$ & 4 & 29 & \\
\hline Biological conduit & $14(15 \%)$ & 0 & 14 & \\
\hline David operation & $4(5 \%)$ & 0 & 4 & \\
\hline $\begin{array}{r}\text { Graft replacement of } \\
\text { ascending aorta }\end{array}$ & & & & NS \\
\hline $\begin{array}{l}\text { Mechanical valve }+ \\
\text { prosthesis }\end{array}$ & $9(10 \%)$ & 0 & 9 & \\
\hline Biological valve + prosthesis & $11(12 \%)$ & 1 & 10 & \\
\hline Prosthesis & $20(22 \%)$ & 7 & 13 & \\
\hline Additional procedure & & & & NS \\
\hline $\begin{array}{l}\text { Coronary artery bypass } \\
\text { surgery }\end{array}$ & $12(13 \%)$ & 3 & 9 & \\
\hline
\end{tabular}

result of extensive and uncontrolled intraoperative hemorrhage at both proximal and distal surgical anastomosis sites. Two of these patients, a 59-year-old man and a 55-year-old woman, developed dehiscent anastomoses, and progression of $\mathrm{AD}$ was macroscopically evident in the remaining aorta. A root operation was then planned, but the patients did not tolerate additional procedures. The third patient with IgG4 positivity was a 20 -year-old man with Marfan syndrome who died during extensive bleeding, but without apparent progression of $\mathrm{AD}$. In addition, one 78-year-old man with IgG4 positivity and severe coronary artery disease died of heart insufficiency shortly after surgery.

A 72-year-old woman with IgG4 negativity died shortly after postoperative mediastinitis and related sepsis. Two patients with $\mathrm{AD}$ and $\mathrm{IgG} 4$ negativity died after hospital discharge, one of whom was a 32-year-old woman with Marfan syndrome who died of unknown reasons after discharge, and one of whom died of extensive liver metastasis associated with rectum carcinoma.

Aortic wall and calculated adventitial inflammation were increased in IgG4-positive aortas compared with IgG4negative aortas $(17.0 \pm 2.1 \mathrm{PSU}$ vs $9.7 \pm 0.6 \mathrm{PSU}, P<$ .001 and $8.7 \pm 0.6 \mathrm{PSU}$ vs $4.7 \pm 0.3 \mathrm{PSU}, P<.001$, respectively). To evaluate whether AD per se had a confounding effect on inflammation, we also analyzed the presence of inflammation after omitting the patients with $\mathrm{AD}$; aortic wall and calculated adventitial inflammation remained increased in IgG4-positive aortas compared with IgG4-negative aortas $(17.5 \pm 3.0 \mathrm{PSU}$ vs $9.4 \pm 0.6 \mathrm{PSU}, P<.001$ and $8.6 \pm 0.9$ PSU vs $4.8 \pm 0.3$ PSU, $P<.001$, respectively). Adventitial inflammation was increased in IgG4-positive aortas compared with IgG4-negative aortas in regard to all investigated inflammatory cells (Table 3, Figure 1). Inflammation and degeneration also were increased in the media in IgG4-positive aortas compared with IgG4-negative aortas. 
TABLE 3. Histology and semiquantitative immunohistochemistry

\begin{tabular}{lcccc}
\hline Mean grade of staining & All patients & IgG4 & IgG4 & $P$ value \\
\hline Adventitia & & & & \\
T cells & $1.6 \pm 0.1$ & $2.3 \pm 0.2$ & $1.4 \pm 0.1$ & $<.001$ \\
B cells & $0.9 \pm 0.1$ & $2.0 \pm 0.3$ & $0.7 \pm 0.1$ & $<.0001$ \\
Macrophages & $1.7 \pm 0.1$ & $2.3 \pm 0.1$ & $1.6 \pm 0.1$ & $<.006$ \\
Plasma cells & $1.1 \pm 0.1$ & $2.1 \pm 0.2$ & $0.9 \pm 0.1$ & $<.0001$ \\
Inflammation & $1.9 \pm 0.1$ & $2.5 \pm 0.2$ & $1.8 \pm 0.1$ & $<.001$ \\
Proliferation & $1.1 \pm 0.1$ & $2.0 \pm 0.3$ & $1.0 \pm 0.1$ & $<.002$ \\
Media & & & & \\
T cells & $0.6 \pm 0.1$ & $0.9 \pm 0.3$ & $0.6 \pm 0.1$ & NS \\
B cells & $0.2 \pm 0.1$ & $0.2 \pm 0.2$ & $0.1 \pm 0.1$ & NS \\
Macrophages & $1.2 \pm 0.1$ & $1.7 \pm 0.3$ & $1.2 \pm 0.1$ & $\mathrm{NS}$ \\
Plasma cells & $0.2 \pm 0.1$ & $0.3 \pm 0.2$ & $0.2 \pm 0.1$ & $\mathrm{NS}$ \\
Inflammation & $1.1 \pm 0.1$ & $1.6 \pm 0.3$ & $1.0 \pm 0.1$ & $<.04$ \\
Proliferation & $1.0 \pm 0.1$ & $1.5 \pm 0.3$ & $1.0 \pm 0.1$ & $\mathrm{NS}$ \\
Degeneration & $1.7 \pm 0.1$ & $2.3 \pm 0.3$ & $1.6 \pm 0.1$ & $<.02$ \\
Elastase & $1.5 \pm 0.1$ & $1.9 \pm 0.4$ & $1.5 \pm 0.1$ & $\mathrm{NS}$ \\
Intima & & & & \\
T cells & $1.2 \pm 0.1$ & $1.9 \pm 0.3$ & $1.2 \pm 0.1$ & $<.009$ \\
B cells & $0.1 \pm 0.1$ & $0.2 \pm 0.2$ & $0.1 \pm 0.1$ & $\mathrm{NS}$ \\
Macrophages & $1.5 \pm 0.1$ & $2.2 \pm 0.2$ & $1.5 \pm 0.1$ & $<.006$ \\
Plasma cells & $0.4 \pm 0.1$ & $0.6 \pm 0.3$ & $0.4 \pm 0.1$ & $\mathrm{NS}$ \\
Inflammation & $1.5 \pm 0.1$ & $1.8 \pm 0.2$ & $1.5 \pm 0.1$ & $\mathrm{NS}$ \\
Proliferation & $0.9 \pm 0.1$ & $1.0 \pm 0.2$ & $0.8 \pm 0.1$ & $\mathrm{NS}$ \\
Thickness & $1.9 \pm 0.1$ & $2.5 \pm 0.3$ & $1.8 \pm 0.1$ & $<.01$ \\
\hline
\end{tabular}

IgG4, Immunoglobulin G4; $N S$, not significant.

Intimal thickness was increased in IgG4-positive aortas compared with IgG4-negative aortas $(2.5 \pm 0.3 \mathrm{PSU}$ and $1.8 \pm 0.1 \mathrm{PSU}$, respectively, $P<.01$ ) because of increased presence of macrophages $(2.2 \pm 0.2 \mathrm{PSU}$ and $1.5 \pm 0.1$ PSU, respectively, $P<.006)$ and T cells $(1.9 \pm 0.3 \mathrm{PSU}$ and $1.2 \pm 0.1 \mathrm{PSU}$, respectively, $P<.009$ ).

\section{Correlation of Aortic Wall and Calculated Adventitial Inflammation}

Aortic wall and calculated adventitial inflammation correlated among all patients $(\mathrm{R} 2$ linear $=0.741, P<.001)$.

\section{Receiver Operating Characteristic Curve Analysis}

The predictive value of IgG4-positive staining to identify aortic wall inflammation was assessed by ROC analysis (Figure 2). IgG4-positive staining was significantly associated with both total aortic wall inflammation (area under the curve [AUC], 0.865; SE, 0.043; $P=.000 ; 95 \%$ confidence interval $[\mathrm{CI}], 0.779-0.950)$ and calculated adventitial inflammation (AUC, 0.912; SE, 0.035; $P=.000 ; 95 \%$ CI, $0.842-0.981$ ). IgG4 positivity was significantly associated with size of the ascending aorta during AAD (AUC, 0.783; SE, 0.102; $P=.004 ; 95 \%$ CI, 0.582-0.984).

\section{DISCUSSION}

Many aortas with AAD were found to be IgG4 positive $(13 \%)$ in our cohort, leaving earlier reports far more modest in reporting the frequency of IgG4-associated aortic disease. Of note, $\mathrm{IgG} 4$ positivity always was confined to the adventitia together with numerous plasma cells and occasional media staining for $\mathrm{IgG} 4$.

There are 3 important clinical messages to be considered. First, IgG4 positivity was not always present during aortitis. Instead, IgG4 positivity together with a distinctive inflammatory pattern including increased plasma and B cells was present in $13 \%$ of all ascending aortas undergoing surgery in our study cohort consisting of consecutive patients. Second, it was confirmed that IgG4 positivity is associated with the likelihood of increased $\mathrm{AAD}$ and $\mathrm{AD}$ compared with IgG4-negative patients. Third, we propose to consider evaluation of the periadventitial tissue during or before the final surgery for the ascending aorta on the basis of the observation that IgG4 positivity always included the adventitia.

Patients with IgG4-positive staining clearly had an increased mean diameter of the ascending aorta at the STJ compared with IgG4-negative patients. Clinically, there is growing fear that an increased aorta diameter may be prone to rupture or $\mathrm{AD}$, and therefore it was no surprise that many patients with IgG4 positivity had AD. Although statistically nonsignificant, approximately half of the patients with IgG4 positivity had AD $(42 \%)$ compared with only 15 of 79 $(19 \%)$ of the remaining patients $(P=.079)$. In addition, 2 IgG4-positive patients underwent immediate surgical reintervention for extensive hemorrhage at the anastomotic sites after completion of the first operation on weaning from cardiopulmonary bypass. For additional interest, 1 more patient underwent operation, having undergone coronary artery bypass operation 3 weeks before diagnosis of $\mathrm{AD}$, which was observed to commence from the proximal anastomosis of the venous graft bypassing the right coronary artery stenosis; this patient also was IgG4 positive. There were 4 deaths $(33 \%)$ in patients with IgG4 positivity and only 3 deaths $(4 \%)$ in patients with IgG4 negativity $(P<$ $.005)$. Only 1 death in both patient groups was unrelated to $\mathrm{AD}$, again emphasizing the fatality of $\mathrm{AD}$.

We believe that the finding in this study of the association of IgG4 positivity with a distinctive inflammatory pattern of aortitis is genuine. Inflammatory focuses per se were not always IgG4 positive. Four of 5 patients with Marfan syndrome were IgG4 negative; the one and only nonspecific vasculitis was IgG4 negative; a preoperative inflammatory state, such as respiratory distress and arthritis, was present in IgG4-positive and negative patients; and 6 of 7 IgG4negative patients had undergone a previous cardiothoracic operation. These findings indirectly suggest that IgG4 positivity may not simply be a measure of inflammation. Only a subset of aortitis may represent aortic manifestation of IgG4-positive aortitis. ${ }^{10}$ Because systematic histopathologic evaluation of the aortic wall revealed increased inflammatory cells of the adventitia in patients with IgG4 positivity, taking into account patients with $\mathrm{AD}$ or without, 
1.
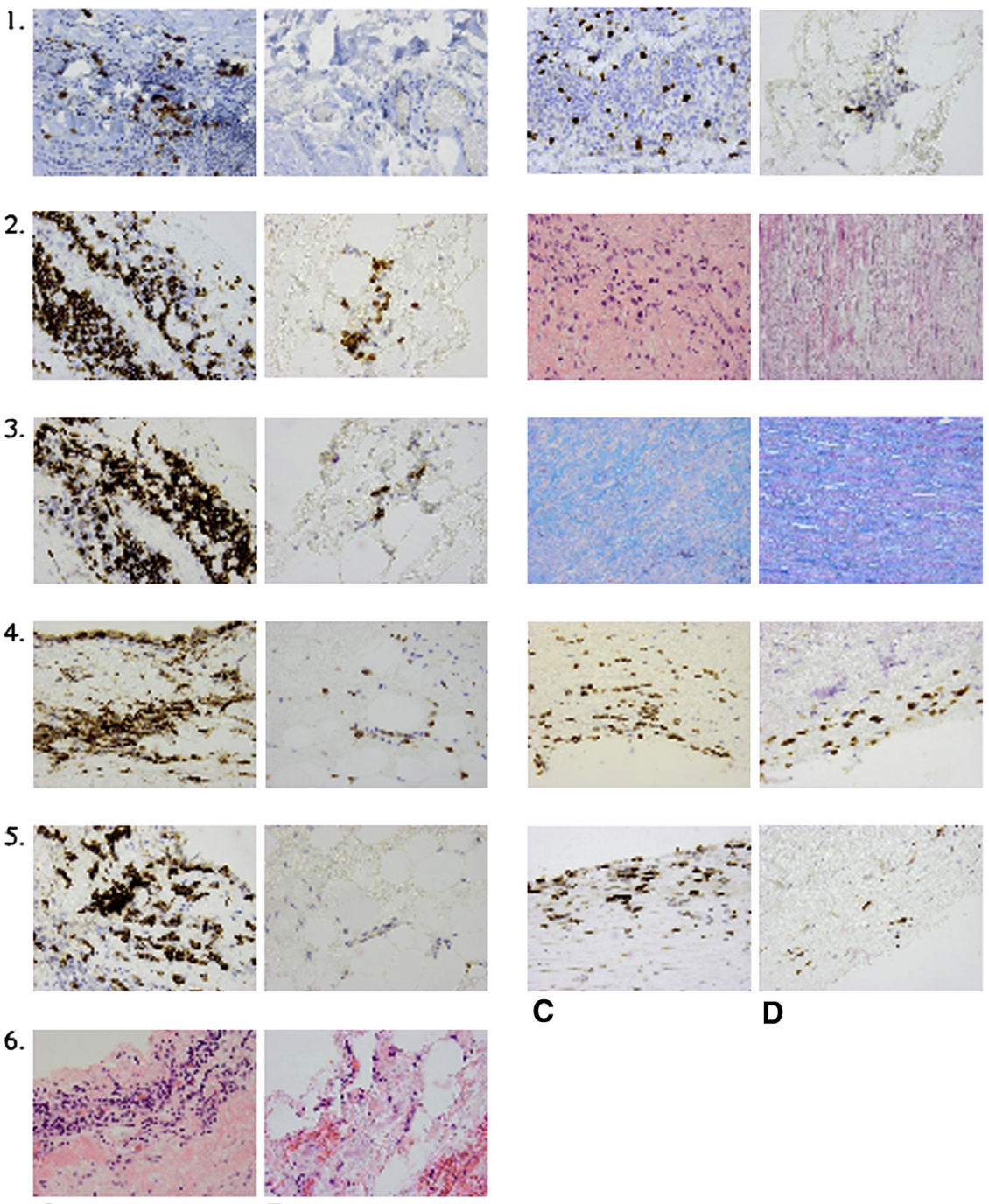

C

D

A

B

FIGURE 1. Representative photographs of aortic wall during AAD $(\times 40)$ showing examples of significant histologic and immunohistochemical parameters, and semiquantitative grading according to PSU. IgG4-positive aorta (A and C) and IgG4-negative aorta (B and D). A and B, left: Row 1: adventitial IgG4 cells. A, PSU 3. B, PSU 0. Row 2: adventitial T cells. A, PSU 3. B, PSU 2. Row 3: adventitial B cells. A, PSU 3. B, PSU 1. Row 4: adventitial macrophages. A, PSU 3. B, PSU 1. Row 5: adventitial plasma cells. A, PSU 3. B, PSU 0. Row 6: adventitial inflammation. A, PSU 3. B, PSU 1. C and D, right: Row 1: medial inflammation. C, PSU 3. D, PSU 0. Row 2: medial degeneration. C, PSU 2. D, PSU 1. Row 3: intimal T cells. C, PSU 3. D, PSU 2. Row 4: intimal macrophages. C, PSU 3. D, PSU 1. Row 5: adventitial proliferation. C, PSU 3. D, PSU 1.

it is tempting to speculate that presence of $\mathrm{IgG} 4$ positivity of the aortic wall is associated with a developing aortitis, increased aortic diameter, and subsequent susceptibility to AD. Calculated adventitial inflammation correlated well with the overall aortic wall inflammation, suggesting that instead of a traditional "inside-out" theory of intima endothelial cell activation and subsequent aortitis, a potential "outside-in" mechanism of adventitial inflammation may be involved. ${ }^{8}$ The overproduction of IgG4-positive plasma cells may be caused by a subset of $T$ cells that attract IgG4-positive B lymphocytes to the adventitia and subsequently differentiate into IgG4-positive plasma cells. ${ }^{15} \mathrm{On}$ the other hand, IgG4-positive B lymphocytes and plasma cells may induce activation of antigen-driven T-helper 2-positive cells that sustain immune response. ${ }^{15}$ Dysregulated T cells may recruit IgG4-positive B cells, subsequently leading to increased IgG4-positive plasma cells that may further differentiate into secretory IgG4 plasma cells. ${ }^{15}$ These secretory IgG4 plasma cells would be responsible for attracting excessive amounts of inflammatory cytokines and growth factors responsible for immunologic activation, as well as excessive remodeling of the periadventitia leading to destruction of aortic wall elasticity. As such, the cause and exact pathophysiology still remain obscure.

IgG4 positivity has been linked to various immunologic conditions, for example, pancreatitis, thyreoiditis, and 


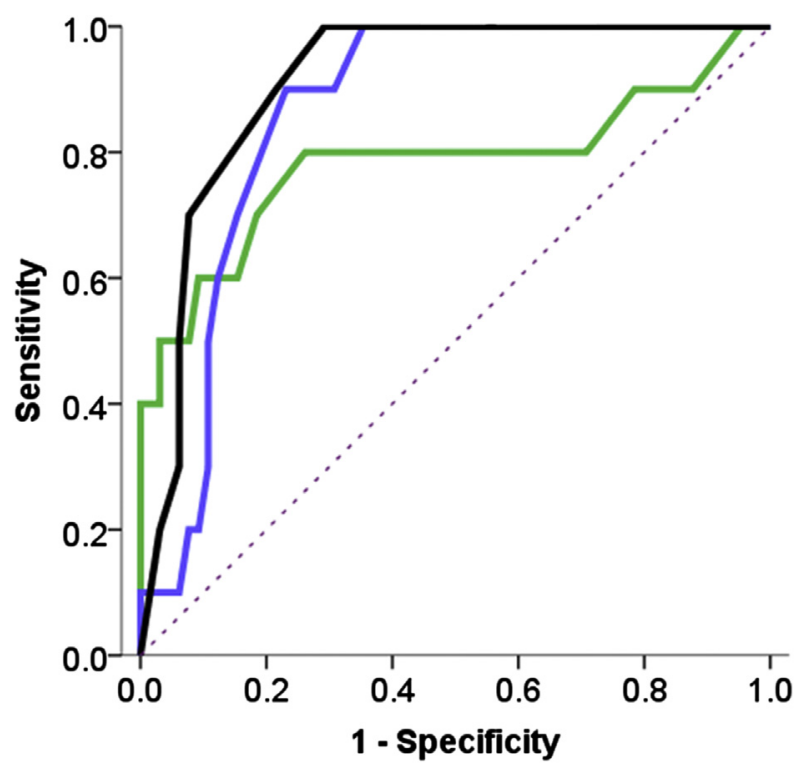

FIGURE 2. ROC analysis of aortic wall IgG4 associated with total aortic wall inflammation (blue line), adventitial inflammation (black line), and $\mathrm{AAD}$ (green line). IgG4 is significantly associated with the prevalence of inflammation (AUC, 0.865; SE, 0.043; $P=.000 ; 95 \%$ CI, 0.779-0.950), calculated adventitial inflammation (AUC, 0.912; SE, 0.035; $P=.000$; 95\% CI, 0.842-0.981), and size of ascending aorta during AAD (AUC, 0.783 ; SE, $0.102 ; P=.004 ; 95 \%$ CI, 0.582-0.984).

tubulointerstitial nephritis. ${ }^{15-17}$ Because numerous IgG4positive plasma cells are found in diverse, localized, nonspecific chronic inflammatory diseases, there is an urgent need to distinguish the clinical relevance of $\mathrm{IgG} 4$ positivity. ${ }^{16}$ In the current study, adventitial plasma cells were elevated in all but 1 patient with IgG4 positivity compared with IgG4-negative aortas. We concede that tissue IgG4 positivity and plasma cell count must be cautiously interpreted within the context of determining IgG4 related systematic disease, as clearly suggested by Strehl and colleagues. ${ }^{16}$ Subsequent studies are warranted to confirm the presence of systematic aortitis together with $\mathrm{IgG} 4$ related positivity. The evaluation of serum $\mathrm{IgG} 4$ may add significance to the presence of tissue $\mathrm{IgG} 4$ positivity. ${ }^{16}$

\section{Study Limitations}

Our study has some limitations. Despite meticulous histologic evaluation, we did not test for IgG4 blood samples, because we did not initially expect to identify such a relatively high number of patients with IgG4-positive aorta $(12 / 91,13 \%)$. We did not perform additional evaluation for IgG4 expression for the residual unresected aorta for the patients who died early after surgery, because experience has not yet been obtained as to whether postmortem histopathology would be comparable to fresh tissue evaluation for IgG4 positivity. One must therefore be cautious with the interpretation of the significance of adventitial
IgG4 positivity in patients with AAD. Our study encompasses only 91 patients, in whom only early follow-up is available. However, IgG4 positivity in our study seems rather frequent in the aortic wall compared with previous reports, and further evaluation of IgG4-related diseases may confirm the association of active aortitis with enlargement of the ascending aorta and risk for AD. Previous studies have investigated for IgG4 positivity only during aortitis. ${ }^{10-13,18}$ In the current study, even small inflammatory focuses were systematically tracked and semiquantified using an armament of inflammatory cell markers, including IgG4 positivity. As previously shown by Laco and colleagues, ${ }^{10}$ microscopic examination of the aorta resected for dilatation is mandatory, because there are often no clinical signs of inflammation, which is evident only at microscopy. In the future, identifying the inflammatory profile of the aorta may take place by means of a rapid immunostaining protocol, similar to the identification of tumor growth and proliferation during surgery ${ }^{19}$ by evaluating the presence of IgG4 positivity of the adventitia.

\section{CONCLUSIONS}

Adventitial IgG4-positive aortitis may enhance susceptibility for AD. Inflammation per se may not solely be of clinical relevance during $\mathrm{AAD}$, and we tentatively challenge the concept that only macroscopic aortitis is of clinical importance. It remains to be investigated whether intervening medically against IgG4 positivity may add an armament against AD.

\section{References}

1. Narayan P, Rogers CA, Davies I, Angelini GD, Bryan AJ. Type A aortic dissection: has surgical outcome improved with time? J Thorac Cardiovasc Surg. 2008; 136:1172-7.

2. Stevens L-M, Madsen JC. Surgical management and long-term outcomes for acute ascending aortic dissection. J Thorac Cardiovasc Surg. 2009;138: 1349-57.

3. Nuenninghoff DM, Hunder GG, Christianson TJ, McClelland RL, Matteson EL. Mortality of large-artery complication (aortic aneurysm, aortic dissection, and/or large-artery stenosis) in patients with giant cell arteritis: a population-based study over 50 years. Arthritis Rheum. 2003;48:3532-7.

4. Pacini D, Leone O, Turci S, Camurri N, Giunchi F, Martinelli GN, et al. Incidence, etiology, histologic findings, and course of thoracic inflammatory aortopathies. Ann Thorac Surg. 2008;86:1518-23.

5. Burke AP, Tavora F, Narula N, Tomaszewski JE, Virmani R. Aortitis and ascending aortic aneurysm: description of 52 cases and proposal of a histologic classification. Hum Pathol. 2008;39:514-26.

6. Collins MJ, Dev V, Strauss BH, Fedak PW, Butany J. Variation in the histopathological features of patients with ascending aortic aneurysms: a study of 111 surgically excised cases. J Clin Pathol. 2008;61:519-23.

7. Homme JL, Aubry MC, Edwards MC, Bagniewski SM, Shane Pankratz V, $\mathrm{Kral} \mathrm{CA}$, et al. Surgical pathology of the ascending aorta: a clinicopathologic study of 513 cases. Am J Surg Pathol. 2006;30:1159-68.

8. He R, Guo DC, Estrera AL, Safi HJ, Huynh TT, Yin Z, et al. Characterization of the inflammatory and apoptotic cells in the aortas of patients with ascending thoracic aortic aneurysms and dissections. J Thorac Cardiovasc Surg. 2006;131: 671-8.

9. Levula M, Paavonen T, Valo T, Pelto-Huikko M, Laaksonen R, Kahonen M, et al. A disintegrin and metalloprotease- 8 and -15 and susceptibility for ascending aortic dissection. Scand J Clin Lab Invest. 2011;71:515-22. 
10. Laco J, Steiner I, Holubec T, Dominik J, Holubcova Z, Vojacek J. Isolated thoracic aortitis: clinicopathological and immunohistochemical study of 11 cases. Cardiovasc Pathol. 2011;20:352-60.

11. Stone JH, Khosroshahi A, Deshpande V, Stone JR. IgG4-related systemic disease accounts for a significant proportion of thoracic lymphoplasmacytic aortitis cases. Arthritis Care Res. 2010;62:316-22.

12. Kasashima S, Zen Y, Kawashima A, Endo M, Matsumoto Y, Kasashima F, et al. A clinicopathologic study of immunoglobulin G4-related sclerosing disease of the thoracic aorta. J Vasc Surg. 2010;52:1587-95.

13. Stone JR. Aortitis, periaortitis, and retroperitoneal fibrosis, as manifestations of IgG4-related systemic disease. Curr Opin Rheumatol. 2011;23:88-94.

14. Elefteriades JA. Thoracic aortic aneurysm: reading the enemy's playbook. World J Surg. 2008;32:366-74
15. Khosroshhi A, Bloch DB, Deshpande V, Stone JH. Rituximab therapy leads to rapid decline of serum IgG4 levels and prompt clinical improvement in IgG4 related systemic disease. Arthritis Rheum. 2010;62:1755-62.

16. Strehl JD, Hartmann A, Agaimy A. Numerous IgG4-positive plasma cells are ubiquitous in diverse localised non-specific chronic inflammatory conditions and need to be distinguished from IgG4-related systemic disorders. J Clin Pathol. 2011;64:237-43.

17. Raissian Y, Nasr SH, Larsen CP, Colvin RB, Smyrk TC, Takahashi N, et al. Diagnosis of IgG4-related tubulointerstitial nephritis. J Am Soc Nephrol. 2011;22:1343-52.

18. Kasashima S, Zen Y. IgG4-related inflammatory abdominal aortic aneurysm. Curr Opin Rheumatol. 2011;23:18-23.

19. Haapasalo J, Mennander A, Helen P, Haapasalo H, Isola J. Ultrarapid Ki-67 immunostaining in frozen section interpretation of gliomas. J Clin Pathol. 2005;58:263-8. 